\title{
Cerebral Microbleeds and Lacunar Infarcts Are Associated with Walking Speed Independent of Cognitive Performance in Middle-Aged to Older Adults
}

\author{
Marjon Stijntjes ${ }^{\mathrm{a}, \mathrm{d}}$ Anton J.M. de Craen ${ }^{\mathrm{a}}$ Jeroen van der Grond ${ }^{\mathrm{b}}$ \\ Carel G.M. Meskers ${ }^{e, f}$ P. Eline Slagboom ${ }^{c}$ Andrea B. Maier ${ }^{f, g}$
}

Departments of ${ }^{\mathrm{a}}$ Gerontology and Geriatrics, ${ }^{\mathrm{b}}$ Radiology and ${ }^{\mathrm{c}}$ Medical Statistics, Section of Molecular Epidemiology, Leiden University Medical Center, Leiden, ${ }^{\mathrm{d} D e p a r t m e n t}$ of Biomechanical Engineering, Delft University of Technology, Delft, e Department of Rehabilitation Medicine, VU University Medical Center, and ${ }^{f}$ MOVE Research Institute Amsterdam, Department of Human Movement Sciences, VU University, Amsterdam, The Netherlands; ${ }^{9}$ Department of Medicine and Aged Care, Royal Melbourne Hospital, University of Melbourne, Melbourne, Vic., Australia

\section{Key Words}

Brain · Hippocampus · Basal ganglia · Cerebral small-vessel disease . Physical performance $\cdot$ Gait $\cdot$ Executive function . Memory

\begin{abstract}
Background: The positive relationship between cognitive and physical performance has been widely established. The influence of brain structure on both domains has been shown as well. Objective: We studied whether the relationship between brain structure and physical performance is independent of cognitive performance. Methods: This was a cross-sectional analysis of 297 middle-aged to older adults (mean age \pm SD $65.4 \pm 6.8$ years). Memory function, executive function and physical performance measured by the Tandem Stance Test, Chair Stand Test, 4-meter walk and 25-meter walk were assessed. Magnetic resonance imaging was available in 237 participants and used to determine the (sub)cortical gray matter, white matter, hippocampal and basal ganglia volumes and the presence of cerebral small-
\end{abstract}

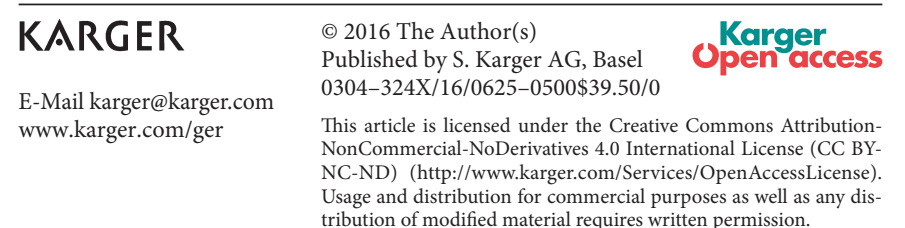

vessel disease, i.e. white matter hyperintensities, cerebral microbleeds (CMBs) and lacunar infarcts (LIs). Regression analysis was used adjusting for age, gender, education and whole-brain volume. A Bonferroni correction was applied considering $\mathrm{p}$ values $<0.017$ as statistically significant. $\boldsymbol{R} \boldsymbol{e}$ sults: Poor memory function was associated with a slower 4-meter walking speed ( $p<0.01$ ). No association was found between brain structure and cognitive performance. The presence of CMBs and LIs was associated with a slower 25 -meter walking speed ( $p<0.001$ ). This result did not change after additional adjustment for cognitive performance. Conclusions: In middle-aged to older adults, CMBs and LIs are associated with walking speed independent of cognitive performance. This emphasizes the clinical relevance of identifying each of the possible underlying mechanisms of physical performance, which is required for the development of timely and targeted therapies.

(c) 2016 The Author(s)

Published by S. Karger AG, Basel

A.J.M.d.C. deceased on January 17, 2016.
Marjon Stijntjes, MSc

Leiden University Medical Center PO Box 9600

NL-2333 RC Leiden (The Netherlands)

E-Mail m.stijntjes@lumc.nl 


\section{Introduction}

The positive relationship between cognitive and physical performance has been widely established. The role of higher-order cognitive performance, especially of executive functioning, in the performance of physical tasks has been shown as well as the influence of the level of physical activity and performance on the risk of dementia and cognitive decline [1-4]. Because of the co-occurrence of cognitive and physical impairment frequently seen with advancing age, a common underlying cause such as brain pathology has been suggested $[5,6]$.

Deterioration of several brain structures has been shown to be associated with cognitive and physical performance. Poor cognitive performance is commonly indicated by brain atrophy and accumulation of white matter hyperintensities (WMHs) $[7,8]$. Thereby, the association of hippocampal volume with global cognitive performance and memory function and of frontal gray matter volumes with executive function has been most consistently shown [9]. Regarding physical performance, especially the association of white matter atrophy and accumulation of WMHs with poor physical performance has been reported [6]. The relation with specific regional and subcortical gray matter volumes is less clear. The few studies incorporating different gray matter volumes showed that especially atrophy of the hippocampus and basal ganglia are associated with poor physical performance $[6,10,11]$.

It is unclear whether the influences of cognitive performance and brain structure on physical performance are independent of each other. In this study, we assessed the relationship between cognitive and physical performance and the relationship of brain structure with both domains within a study population of relatively healthy middle-aged to older adults in the absence of overt diseases.

\section{Methods}

\section{Leiden Longevity Study}

The Leiden Longevity Study is a longitudinal cohort comprising of 421 long-lived Caucasian families of Dutch descent recruited between 2002 and 2006 [12]. Families were enrolled if at least two long-lived siblings were alive and fulfilled the age criterion of 89 years or older for males and 91 years or older for females. Additionally, the offspring of these long-lived siblings were included together with their partners as controls representing the general population [13]. For the current study, participants were recruited from this population of middle-aged to older adults visiting the research center during the follow-up measurement between Sep- tember 2009 and December 2010. Participants were included if they had complete data on both cognitive and physical performance leading to 297 participants (149 offspring and 148 partners). The Medical Ethical Committee of the Leiden University Medical Center approved the study, and written informed consent was obtained from all participants.

\section{Cognitive Performance}

Cognitive performance was tested for two different cognitive domains: memory and executive function. Memory function was assessed by the immediate and delayed recall of the 15-Picture Learning Test (15-PLTi and 15-PLTd, respectively). For the immediate recall, 15 pictures of well-known items were shown to the participant at three consecutive times. After each time, the participant was asked to recall as many pictures as possible. The sum of the number of correct pictures during the three trials was defined as the immediate recall. The delayed recall was defined as the number of correct pictures the participant was able to recall after 20 min.

Executive function was assessed by attention and processing speed using the abbreviated Stroop Test Trial 3 and the Digit Symbol Substitution Test (DSST), respectively. The abbreviated Stroop Test Trial 3 consisted of a card containing 40 color words printed in a discongruous ink color. The participant was asked to name the ink color of the words as fast as possible. In the DSST, digits were given on a card as well as a key for the formation of specific digitsymbol combinations. The outcome parameter was the number of correct digit-symbol combinations the participant was able to make in $90 \mathrm{~s}$.

\section{Physical Performance}

Four different aspects of physical performance were assessed: the ability to maintain standing balance, the ability to rise from a chair, and walking speed over a short and long distance $[14,15]$. The ability to maintain standing balance was assessed by the Tandem Stance Test. Participants were asked to place their feet in tandem position, i.e. placing both feet in line with one foot touching the toe of the other foot, and maintaining balance for $10 \mathrm{~s}$. The Chair Stand Test was used to assess the ability to rise from a chair. Participants were asked to stand up and sit down 5 times as quickly as possible from a straight-backed chair. The test was started in the sitting position and ended when the participant was standing with a straight back for the 5th time. Walking speed was assessed over 4 and $25 \mathrm{~m}$ of walking. During the 4-Meter Walking Test, participants started from the standing position and were instructed to walk at their preferred speed over a length of $5 \mathrm{~m}$ without slowing down before the 4-meter line. Time was started at the moment the first foot had passed the starting line until the moment the first foot had passed the 4-meter line completely. The fastest time out of two measurements was used for the calculation of walking speed. Walking speed during $25 \mathrm{~m}$ of walking was assessed in a steady state over a length of $30 \mathrm{~m}$, i.e. time was started at $2.5 \mathrm{~m}$ and stopped at $27.5 \mathrm{~m}$. Again, participants were asked to walk at their preferred speed. Mean walking speed over two measurements was used for analyses.

During all physical performance tests, participants wore nonslip socks and a body-fixed sensor (Dynaport ${ }^{\circledR}$ Hybrid, McRoberts BV, The Hague, the Netherlands) on the lower back positioned with an elastic belt at the height of the second lumbar vertebra. The sensor consisted of a triaxial accelerometer and three uniaxial gy- 
roscopes of which the signals were recorded with a sample frequency of $100 \mathrm{~Hz}$. A remote control was used for marking the start and end of each measurement.

\section{Brain Structure}

Magnetic resonance imaging (MRI) was used to determine the whole-brain volume, the cortical gray and white matter volumes, the subcortical gray matter volumes of the hippocampus and basal ganglia, including the nucleus accumbens, caudate nucleus, pallidum and putamen, and the presence of cerebral small-vessel disease including $\mathrm{WMH}$ volume and the presence of cerebral microbleeds (CMBs) and lacunar infarcts (LIs) [16, 17]. Threedimensional (3-D) T1-weighted, T2-weighted, fluid-attenuated inversion recovery (FLAIR) and T2*-weighted images were acquired using a whole-body MR system operating at a field strength of $3 \mathrm{~T}$ (Philips Medical Systems, Best, The Netherlands) with the following imaging parameters: repetition time $9.7 \mathrm{~ms}$, time to echo $4.6 \mathrm{~ms}$, flip angle $8^{\circ}$ and field of view $224 \times 177 \times 168 \mathrm{~mm}$ resulting in a normal voxel size of $1.17 \times 1.17 \times 1.4 \mathrm{~mm}$.

Different tools of the FMRIB Software Library [FSL, Release 5.0 (c) 2012] [18, 19] were used for the determination of brain volumes. Whole brain, gray matter and white matter volumes were calculated with the FSL-Tool Structural Image Evaluation, using Normalization of Atrophy (SIENAX) [20, 21]. For the determination of the subcortical hippocampus and basal ganglia volumes, the FMRIB's Registration and Segmentation Tool (FIRST) was used.

MRI scans were visualized using the freely available software Medical Imaging Processing, Analysis and Visualization (MIPAV). WMHs, CMBs and LIs were analyzed by two independent reviewers who were blinded to subject identity, sex, age and being offspring or partner. Discrepancies were reanalyzed in a consensus meeting with a third reviewer. WMHs were defined as areas within the cerebral white matter with increased signal intensity on both FLAIR and T2*-weighted images without mass effect. CMBs were defined as focal areas of signal void on T2-weighted images increasing in size on $\mathrm{T} 2{ }^{*}$-weighted images. The presence of LIs, defined as having a diameter $>2 \mathrm{~mm}$, was assessed on 3-D T1-weighted, FLAIR and T2-weighted images. The presence of CMBs and LIs was combined into one variable consisting of participants with either CMBs or LIs or both. Due to contraindications for MRI, data were available for 237 out of 297 (80\%) participants. The most frequently reported contraindications were metal splinters, pacemaker and claustrophobia as determined by applying the standard MRI patient questionnaire of the Leiden University Medical Center.

\section{Statistical Analysis}

Continuous variables are presented as means and standard deviations, non-Gaussian distributed variables as medians and interquartile ranges, and categorical variables as numbers and percentages.

First, the association between the cognitive and physical performance was assessed using linear regression analysis. Logistic regression analysis was carried out for the dichotomous outcome of the Tandem Stance Test, i.e. participants being either unable or able to maintain $10 \mathrm{~s}$ of balance in a tandem position. Results were adjusted for age, gender and education.

Second, linear regression analysis was used to assess the relationship between brain structure and cognitive performance and subsequently between brain structure and physical performance. Again, logistic regression analysis was carried out for the Tandem
Table 1. Characteristics of the participants $(n=297)$

\begin{tabular}{|c|c|}
\hline \multicolumn{2}{|l|}{ Demographics } \\
\hline Females, n (\%) & $150(50.5)$ \\
\hline Age, years & $65.4(6.8)$ \\
\hline Education, years & $12(10-15)$ \\
\hline \multicolumn{2}{|l|}{ Anthropometrics } \\
\hline Height, $\mathrm{cm}$ & $172.4(8.5)$ \\
\hline Weight, kg & $79.2(14.1)$ \\
\hline \multicolumn{2}{|l|}{ Comorbidities $^{c}$} \\
\hline Diabetes mellitus, n (\%) & $18(6.8)$ \\
\hline Cardiovascular disease $^{\mathrm{a}}, \mathrm{n}(\%)$ & $79(29.8)$ \\
\hline \multicolumn{2}{|l|}{ Intoxications } \\
\hline Users of alcohol ${ }^{\mathrm{b}}, \mathrm{n}(\%)$ & $234(79.1)$ \\
\hline Former and/or current smokers, n (\%) & $201(67.9)$ \\
\hline \multicolumn{2}{|l|}{ Cognitive performance } \\
\hline 15-PLTi, correct pictures & $30.1(5.0)$ \\
\hline 15-PLTd, correct pictures & $11(10-13)$ \\
\hline Stroop time part $3, \mathrm{~s}$ & $46(39-54)$ \\
\hline DSST, correct answers & $47.1(10.6)$ \\
\hline \multicolumn{2}{|l|}{ Physical performance } \\
\hline Able to perform tandem stance, n (\%) & $286(96.6)$ \\
\hline Duration of the CST, s & $12.6(2.4)$ \\
\hline 4-meter walking speed, m/s & $1.13(0.20)$ \\
\hline 25-meter walking speed ${ }^{\mathrm{d}}, \mathrm{m} / \mathrm{s}$ & $1.45(0.16)$ \\
\hline \multicolumn{2}{|l|}{ Brain structure } \\
\hline Whole-brain volume $\mathrm{em}^{\mathrm{e}}$ & $1,090(92)$ \\
\hline Gray matter volume $\mathrm{e}^{\mathrm{e}} \mathrm{cm}^{3}$ & $542(39)$ \\
\hline White matter volume $\mathrm{e}^{\mathrm{e}} \mathrm{cm}^{3}$ & $548(61)$ \\
\hline Hippocampal volume ${ }^{\mathrm{e}}, \mathrm{cm}^{3}$ & $8.0(0.8)$ \\
\hline Basal ganglia volume $\mathrm{e}^{\mathrm{e}}, \mathrm{cm}^{3}$ & $21.8(2.2)$ \\
\hline WMH volume ${ }^{\mathrm{f}}, \mathrm{cm}^{3}$ & $0.5(0.083-2.2)$ \\
\hline Presence of CMBs/LIs ${ }^{\mathrm{e}}, \mathrm{n}(\%)$ & $37(15.7)$ \\
\hline
\end{tabular}

Values are expressed as mean (standard deviation), median (interquartile range) or as stated. ${ }^{\mathrm{a}}$ Defined as myocardial infarction, stroke and hypertension. ${ }^{\mathrm{b}}$ Using $\geq 1$ units per week. Data available for: ${ }^{\mathrm{c}} \mathrm{n}=265,{ }^{\mathrm{d}} \mathrm{n}=277,{ }^{\mathrm{e}} \mathrm{n}=237$, and ${ }^{\mathrm{f}} \mathrm{n}=211$ participants.

Stance Test. All measures of brain structure were expressed as Z scores. For the basal ganglia volume, a composite $\mathrm{Z}$ score was calculated by averaging the $Z$ scores of the nucleus accumbens, caudate nucleus, pallidum and putamen. Results were adjusted for age, gender and whole-brain volume. An additional adjustment model was used in the analysis of the relationship between brain structure and physical performance including cognitive performance besides age, gender and whole-brain volume. For this analysis, a composite $\mathrm{Z}$ score of cognitive performance was calculated by averaging the $Z$ scores of the 15-PLTi, 15-PLTd, abbreviated Stroop Test Trial 3 (multiplied by -1 because of the reverse direction of scoring compared to the other cognitive performance tests) and DSST.

The independent $t$ test and $\chi^{2}$ test were used to assess whether the characteristics of the participants with and without MRI data 
Table 2. Association between cognitive and physical performance $(n=297)$

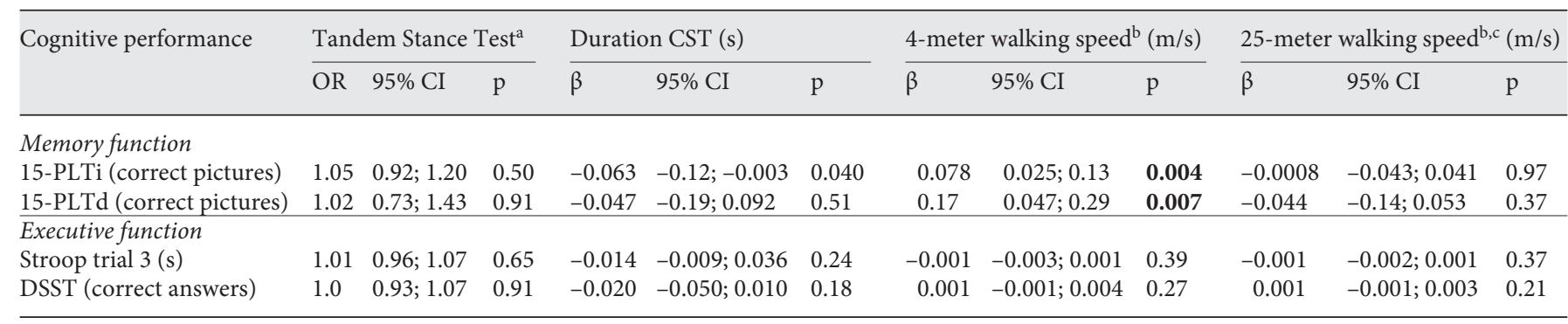

$\mathrm{OR}=$ Odds ratio $\mathrm{CI}=$ confidence interval; $\beta=$ estimate. $\mathrm{CST}=$ Chair Stand Test. Associations were assessed using linear regression adjusted for calendar age, gender and education. Bold indicates statistically significant $\mathrm{p}$ values after Bonferroni correction $(\mathrm{p}<0.017)$. ${ }^{\mathrm{a}}$ Logistic regression was used with $0=$ unable and $1=$ able to perform the Tandem Stance Test. ${ }^{b} \beta$ and $95 \%$ CI are expressed per 10 units of cognitive performance test. ${ }^{c} n=277$.

Table 3. Association between brain structure and cognitive performance $(n=237)$

\begin{tabular}{|c|c|c|c|c|c|c|c|c|c|c|c|c|}
\hline \multirow[t]{2}{*}{ Brain structure $^{a}$} & \multicolumn{3}{|c|}{ 15-PLTi (correct pictures) } & \multicolumn{3}{|c|}{ 15-PLTd (correct pictures) } & \multicolumn{3}{|c|}{ Stroop trial $3(\mathrm{~s})$} & \multicolumn{3}{|c|}{ DSST (correct answers) } \\
\hline & $\beta$ & $95 \%$ CI & $\mathrm{p}$ & $\beta$ & $95 \%$ CI & $\mathrm{p}$ & $\beta$ & $95 \%$ CI & $\mathrm{p}$ & $\beta$ & $95 \%$ CI & $\mathrm{p}$ \\
\hline \multicolumn{13}{|l|}{ Cortical } \\
\hline Gray matter volume & 0.75 & $-0.39 ; 1.89$ & 0.20 & -0.13 & $-0.63 ; 0.38$ & 0.63 & 0.70 & $-2.42 ; 3.83$ & 0.66 & -0.41 & $-2.88 ; 2.05$ & 0.74 \\
\hline White matter volume & -0.71 & $-1.69 ; 0.27$ & 0.15 & 0.11 & $-0.32 ; 0.54$ & 0.62 & -0.85 & $-3.54 ; 1.84$ & 0.53 & 0.36 & $-1.76 ; 2.48$ & 0.74 \\
\hline \multicolumn{13}{|l|}{ Subcortical } \\
\hline Hippocampal volume & -0.064 & $-0.76 ; 0.63$ & 0.86 & -0.13 & $-0.43 ; 0.17$ & 0.40 & -1.31 & $-3.19 ; 0.58$ & 0.17 & -0.42 & $-1.90 ; 1.07$ & 0.58 \\
\hline Basal ganglia volume & -0.26 & $-0.94 ; 0.43$ & 0.47 & -0.22 & $-0.52 ; 0.075$ & 0.14 & 0.31 & $-1.58 ; 2.20$ & 0.75 & -0.84 & $-2.34 ; 0.66$ & 0.27 \\
\hline \multicolumn{13}{|l|}{ CSVD } \\
\hline WMH volume $\mathrm{b}^{\mathrm{b}}$ & -0.037 & $-0.71 ; 0.64$ & 0.91 & -0.021 & $-0.32 ; 0.28$ & 0.89 & 0.72 & $-1.10 ; 2.36$ & 0.44 & -0.37 & $-1.77 ; 1.04$ & 0.61 \\
\hline Presence of CMBs/LIs ${ }^{c}$ & -0.16 & $-1.73 ; 1.42$ & 0.84 & 0.31 & $-0.38 ; 1.0$ & 0.38 & -1.72 & $-6.04 ; 2.59$ & 0.43 & 2.78 & $-0.62 ; 6.18$ & 0.11 \\
\hline
\end{tabular}

$\mathrm{p}$ values $<0.017$ are considered as statistically significant after Bonferroni correction. $\beta=$ Estimate; $\mathrm{CI}=$ confidence interval; $\mathrm{CSVD}=$ cerebral small-vessel disease. Associations were assessed using linear regression adjusted for age, gender and whole-brain volume (standardized for skull size). ${ }^{\text {a }}$ Brain volumes are standardized for skull size and expressed as $\mathrm{Z}$ scores. WMH volume is transformed to the natural logarithm and expressed as $\mathrm{Z}$ score. ${ }^{\mathrm{b}} \mathrm{n}=211 .{ }^{\mathrm{c}} 0$ is defined as reference group having no CMBs or no LIs.

were significantly different. Because of the number of dependent variables within each of the comparisons that were investigated, i.e. the relationship between cognitive and physical performance, brain structure and cognitive performance and brain structure and physical performance, a Bonferroni correction was applied to avoid type I errors. p values $<0.017$ were considered statistically significant. All statistical analyses were performed with SPSS version 22.0 (IBM Corp., Amonk, N.Y., USA).

\section{Results}

Characteristics of the participants are given in table 1. Mean age \pm SD of all participants was $65.4 \pm 6.8$ years. Table 2 shows the association between cognitive and physical performance. According to memory function, participants with a worse performance on the immediate and delayed recall had a slower 4-meter walking speed.
No associations were found for executive function or other physical domains.

Tables 3 and 4 present the association between brain structure and cognitive and physical performance, respectively. No association was found between brain structure and cognitive performance. According to the different domains of physical performance, the presence of CMBs and LIs was associated with a slower 25-meter walking speed.

Table 5 shows that additional adjustment for cognitive performance in the association between brain structure and physical performance did not change the results. There were no differences between the participants with and without MRI data, except for weight and the prevalence of cardiovascular disease, which were both higher in the group without MRI data $(\mathrm{p}=0.026$ and $\mathrm{p}=0.021$, respectively). 
Table 4. Association between brain structure and physical performance $(n=237)$

\begin{tabular}{|c|c|c|c|c|c|c|c|c|c|c|c|c|}
\hline \multirow[t]{2}{*}{ Brain structure $^{a}$} & \multicolumn{3}{|c|}{ Tandem Stance Test ${ }^{\mathrm{b}}$} & \multicolumn{3}{|c|}{ Duration CST (s) } & \multicolumn{3}{|c|}{ 4-meter walking speed $(\mathrm{m} / \mathrm{s})$} & \multicolumn{3}{|c|}{25 -meter walking speed $(\mathrm{m} / \mathrm{s})$} \\
\hline & OR & $95 \%$ CI & $\mathrm{p}$ & $\beta$ & $95 \% \mathrm{CI}$ & $\mathrm{p}$ & $\beta$ & $95 \%$ CI & $\mathrm{p}$ & $\beta$ & $95 \% \mathrm{CI}$ & $\mathrm{p}$ \\
\hline \multicolumn{13}{|l|}{ Cortical } \\
\hline Gray matter volume & 2.21 & $0.48 ; 10.2$ & 0.31 & -0.10 & $-0.70 ; 0.49$ & 0.73 & 0.022 & $-0.029 ; 0.073$ & 0.40 & 0.030 & $-0.010 ; 0.069$ & 0.15 \\
\hline White matter volume & 0.34 & $0.10 ; 1.10$ & 0.072 & 0.13 & $-0.38 ; 0.64$ & 0.62 & -0.019 & $-0.062 ; 0.025$ & 0.40 & -0.028 & $-0.062 ; 0.007$ & 0.11 \\
\hline \multicolumn{13}{|l|}{ Subcortical } \\
\hline Hippocampal volume & 2.36 & $0.96 ; 5.84$ & 0.062 & -0.30 & $-0.66 ; 0.066$ & 0.11 & 0.033 & $0.002 ; 0.063$ & 0.035 & 0.010 & $-0.015 ; 0.034$ & 0.43 \\
\hline Basal ganglia volume & 1.40 & $0.60 ; 3.27$ & 0.44 & 0.11 & $-0.25 ; 0.47$ & 0.54 & -0.017 & $-0.047 ; 0.014$ & 0.29 & -0.015 & $-0.039 ; 0.009$ & 0.21 \\
\hline \multicolumn{13}{|l|}{ CSVD } \\
\hline WMH volume $e^{c}$ & 1.85 & $0.89 ; 3.82$ & 0.098 & 0.013 & $-0.32 ; 0.35$ & 0.94 & 0.005 & $-0.024 ; 0.034$ & 0.73 & -0.023 & $-0.045 ;-0.0002$ & 0.048 \\
\hline Presence of CMBs/LIs ${ }^{\mathrm{d}}$ & 2.98 & $0.29 ; 30.3$ & 0.36 & -0.039 & $-0.85 ; 0.78$ & 0.93 & -0.080 & $-0.15 ;-0.011$ & 0.024 & -0.11 & $-0.17 ;-0.060$ & $<0.001$ \\
\hline
\end{tabular}

$\mathrm{OR}=$ Odds ratio $\mathrm{CI}=$ confidence interval; $\beta=$ estimate $\mathrm{CSVD}=$ cerebral small-vessel disease. Associations were assessed using linear regression adjusted for age, gender and whole-brain volume (standardized for skull size). Bold indicates statistically significant $\mathrm{p}$ values after Bonferroni correction ( $\mathrm{p}<0.017)$. a Brain volumes are standardized for skull size and expressed as $\mathrm{Z}$ scores. WMH volume is transformed to the natural logarithm and expressed as $\mathrm{Z}$ score. ${ }^{\mathrm{b}}$ Logistic regression was used with $0=$ unable and $1=$ able to perform the Tandem Stance Test. ${ }^{\mathrm{c}} \mathrm{n}=211 .{ }^{\mathrm{d}} 0$ is defined as reference group having no CMBs or no LIs. ${ }^{\mathrm{e}} \mathrm{n}=220$.

Table 5. Association between brain structure and physical performance, adjusted additionally for cognitive performance $(\mathrm{n}=237)$

\begin{tabular}{|c|c|c|c|c|c|c|c|c|c|c|c|c|}
\hline \multirow[t]{2}{*}{ Brain structure $^{a}$} & \multicolumn{3}{|c|}{ Tandem Stance Test ${ }^{\mathrm{b}}$} & \multicolumn{3}{|c|}{ Duration CST (s) } & \multicolumn{3}{|c|}{ 4-meter walking speed $(\mathrm{m} / \mathrm{s})$} & \multicolumn{3}{|c|}{25 -meter walking speed $(\mathrm{m} / \mathrm{s})$} \\
\hline & OR & $95 \%$ CI & $\mathrm{p}$ & $\beta$ & $95 \%$ CI & $\mathrm{p}$ & $\beta$ & $95 \% \mathrm{CI}$ & $\mathrm{p}$ & $\beta$ & $95 \%$ CI & $\mathrm{p}$ \\
\hline \multicolumn{13}{|l|}{ Cortical } \\
\hline Gray matter volume & 2.25 & $0.49 ; 10.2$ & 0.30 & -0.10 & $-0.69 ; 0.49$ & 0.74 & 0.023 & $-0.028 ; 0.074$ & 0.37 & 0.030 & $-0.010 ; 0.069$ & 0.15 \\
\hline White matter volume & 0.33 & $0.10 ; 1.08$ & 0.67 & 0.13 & $-0.38 ; 0.64$ & 0.63 & -0.020 & $-0.063 ; 0.024$ & 0.38 & -0.028 & $-0.062 ; 0.007$ & 0.11 \\
\hline \multicolumn{13}{|l|}{ Subcortical } \\
\hline Hippocampal volume & 2.39 & $0.96 ; 5.95$ & 0.062 & -0.30 & $-0.66 ; 0.064$ & 0.11 & 0.033 & $0.003 ; 0.063$ & 0.034 & 0.010 & $-0.015 ; 0.034$ & 0.43 \\
\hline Basal ganglia volume & 1.42 & $0.61 ; 3.34$ & 0.42 & 0.086 & $-0.27 ; 0.44$ & 0.64 & -0.015 & $-0.045 ; 0.016$ & 0.35 & -0.015 & $-0.039 ; 0.009$ & 0.21 \\
\hline \multicolumn{13}{|l|}{ CSVD } \\
\hline WMH volume ${ }^{c}$ & 1.88 & $0.90 ; 3.94$ & 0.094 & 0.006 & $-0.33 ; 0.34$ & 0.97 & 0.006 & $-0.023 ; 0.034$ & 0.69 & -0.023 & $-0.046 ;-0.0002$ & 0.048 \\
\hline Presence of CMBs/LIs ${ }^{\mathrm{d}}$ & 2.93 & $0.29 ; 30.1$ & 0.37 & 0.004 & $-0.81 ; 0.82$ & 0.99 & -0.084 & $-0.15 ;-0.015$ & 0.017 & -0.11 & $-0.17 ;-0.061$ & $<0.001$ \\
\hline
\end{tabular}

Bold indicates statistically significant $\mathrm{p}$ values after Bonferroni correction $(\mathrm{p}<0.017) . \mathrm{OR}=$ Odds ratio; $\mathrm{CI}=$ confidence interval; $\beta=$ estimate; CST $=$ Chair Stand Test; CSVD = cerebral small vessel disease. Associations were assessed using linear regression adjusted for age, gender, whole-brain volume (standardized for skull size) and cognitive performance expressed as composite Z score. ${ }^{\text {a }}$ Brain volumes are standardized for skull size and expressed as Z scores. WMH volume is transformed to the natural logarithm and expressed as Z score. ${ }^{\mathrm{b}}$ Logistic regression was used with $0=$ unable and $1=$ able to perform the tandem stance. ${ }^{c} n=211 .{ }^{d} 0$ is defined as reference group having no CMBs or no LIs. ${ }^{e} n=220$.

\section{Discussion}

In this study population of middle-aged to older adults, poor memory function was associated with slower 4-meter walking speed. The presence of CMBs and LIs was associated with slower 25 -meter walking speed. No association was found between brain structure and cognitive performance, and the relationship between brain structure and physical performance did not change after adjustment for cognitive performance.

The association between cognitive and physical performance has been confirmed by several studies $[1,2,22$, 23]. However, these studies especially show the role of executive function in gait performance commonly illustrated by the performance of dual tasks [1, 24-27]. The association between memory function and physical performance has been shown less frequently, although the incorporation of memory processes in physical performance has previously been emphasized as well $[11,28$, 29 ]. The association of memory function with the 4-meter walking speed, and not with the 25 -meter walking speed, suggests that specifically the initiation of movements relates to memory processes. In the 4-meter walk, walking speed was measured from the starting position including the initiation phase instead of the steady state assessment of walking speed in the 25 -meter walk. These 
findings are supported by a previous study in elderly outpatients, showing largest effect sizes of the association between cognitive performance and walking speed for the 4-meter walk compared to a steady-state 10 -meter walk and a 6-min walk [30].

Among the different physical domains that were assessed, i.e. the ability to maintain standing balance, the ability to rise from a chair and walking speed over a short and long distance, brain structure was specifically associated with walking speed. This emphasizes the role of walking speed as an important indicator of overall health status [31, 32]. Of the two different walking tests that were performed, the 25-meter walking speed, and not the 4-meter walking speed, was associated with brain structure, i.e. the presence of CMBs and LIs. This specific association might be explained by the dependence of longer distance walks on the cardiovascular determined endurance factor, considering that the presence of CMBs and LIs is accelerated by cardiovascular disease and vascular risk factors [30, 33]. Besides the consistent associations of cortical and subcortical brain volumes with walking speed found in the literature [6], no association was found for each of the brain volumes in this study. These results might be explained by differences in calendar age between the study populations. Most studies showing a positive association between brain structure and walking speed are in slightly older populations (mean age of 70 years and older) [6], while the null results of this study are in accordance with a previous study in a comparable population of healthy middle-aged to older adults walking at preferred speed over a long distance [34].

The relationship between brain structure and physical performance did not change after adjustment for cognitive performance, which is consistent with the fact that we did not find an association between brain structure and cognitive performance. Previously, no association of measures of brain microstructure, i.e. mean magnetization transfer ratio, mean magnetization transfer ratio histogram peak height, fractional anisotropy and mean diffusivity, with cognitive performance was found in this study population [35]. The relatively young age might explain these results if we compare them with the positive associations found in studies in older communitydwelling individuals without cognitive impairment [9, $36,37]$. Thereby, the measures used for the assessment of the relation between brain structure and cognitive performance might not be sensitive enough for this population [9]. Future studies focusing on which measures are most sensitive for these nonclinical populations might provide more insight into this. The association of the presence of CMBs and LIs with a slower 25-meter walking speed independent of cognitive performance suggests that the influences of brain structure and cognitive performance on physical performance are two independent processes. This is supported by the positive association found between memory function and 4-meter walking speed, while no association was found of the hippocampal volume, known to play an important role in memory function $[9,38]$, with the 4-meter walking speed. These results, therefore, emphasize the importance of identifying possible underlying mechanisms of physical performance in order to be able to develop targeted therapies. Longitudinal studies covering a broader age range are needed to further explore this and to finally get insight into the optimal timing with respect to prevention and disease progression.

One of the strengths of this study is the availability of data on cognitive and physical performance and for $80 \%$ of the participants on brain structure, with all measurements of one participant performed on the same day. This enabled us to get insight into the role of cognitive performance and brain structure on physical performance. The different cognitive and physical domains that were assessed enabled to specify this even further. The study population, consisting of middle-aged to older adults, enhanced our understanding of changes that are already observable from middle age in the absence of overt diseases. As a result of this age range and good health status of the participants, the variation among participants able and unable to perform the Tandem Stance Test was very low. This could have led to an underestimation of the associations of cognitive performance and brain structure with standing balance. Future studies are needed including a more demanding standing condition, for example with eyes closed, to further investigate the association of cognitive performance and brain structure with standing balance. Another limitation is the availability of MRI data in $80 \%$ of the participants due to contraindications for MRI. The higher prevalence of cardiovascular disease in the group of participants without MRI data is in concordance with our expectations because of the contraindications for MRI. Exclusion of these participants could have led to an underestimation of the results, specifically for the association of the presence of cerebral small-vessel disease with the 25-meter walking speed.

In conclusion, the presence of CMBs and LIs is associated with walking speed in middle-aged to older adults, independent of cognitive performance. This supports 
that in this age range the influences of brain structure and cognitive performance on physical performance are two independent processes. Identifying the possible influence of these underlying mechanisms of physical performance is of clinical relevance in order to be able to develop targeted therapies with optimal timing to maintain physical performance with advancing age.

\section{Acknowledgements}

This work was supported by a grant from the Netherlands Genomics Initiative/Netherlands Organization for scientific research [NGI/NWO; 05040202 and 050-060-810 Netherlands Consortium for Healthy Aging (NCHA)] and the seventh framework program MYOAGE (HEALTH-2007-2.4.5-10).

\section{Disclosure Statement}

None of the authors has any conflicts of interest to declare.

\section{References}

1 Sheridan PL, Hausdorff JM: The role of higher-level cognitive function in gait: executive dysfunction contributes to fall risk in $\mathrm{Alz}$ heimer's disease. Dement Geriatr Cogn Disord 2007;24:125-137.

2 Clouston SA, Brewster P, Kuh D, Richards M, Cooper R, Hardy R, Rubin MS, Hofer SM: The dynamic relationship between physical function and cognition in longitudinal aging cohorts. Epidemiol Rev 2013;35:33-50.

-3 Sofi F, Valecchi D, Bacci D, Abbate R, Gensini GF, Casini A, Macchi C: Physical activity and risk of cognitive decline: a meta-analysis of prospective studies. J Intern Med 2011;269: 107-117.

4 Wang L, Larson EB, Bowen JD, van Belle G: Performance-based physical function and future dementia in older people. Arch Intern Med 2006;166:1115-1120.

-5 Buchman AS, Yu L, Wilson RS, Boyle PA, Schneider JA, Bennett DA: Brain pathology contributes to simultaneous change in physical frailty and cognition in old age. J Gerontol A Biol Sci Med Sci 2014;69:1536-1544.

6 Kilgour AH, Todd OM, Starr JM: A systematic review of the evidence that brain structure is related to muscle structure and their relationship to brain and muscle function in humans over the lifecourse. BMC Geriatr 2014; 14:85.

7 Fotenos AF, Snyder AZ, Girton LE, Morris JC, Buckner RL: Normative estimates of cross-sectional and longitudinal brain volume decline in aging and AD. Neurology 2005;64:1032-1039.

8 Prins ND, Scheltens P: White matter hyperintensities, cognitive impairment and dementia: an update. Nat Rev Neurol 2015;11:157165.

9 Kaup AR, Mirzakhanian H, Jeste DV, Eyler LT: A review of the brain structure correlates of successful cognitive aging. J Neuropsychiatry Clin Neurosci 2011;23:6-15.

10 Dumurgier J, Crivello F, Mazoyer B, Ahmed I, Tavernier B, Grabli D, Francois C, TzourioMazoyer N, Tzourio C, Elbaz A: MRI atrophy of the caudate nucleus and slower walking speed in the elderly. Neuroimage 2012;60: 871-878.
-11 Scherder E, Eggermont L, Swaab D, van Heuvelen M, Kamsma Y, de Greef M, van Wijck R, Mulder T: Gait in ageing and associated dementias; its relationship with cognition. Neurosci Biobehav Rev 2007;31:485-497.

12 Schoenmaker M, de Craen AJ, de Meijer PH, Beekman M, Blauw GJ, Slagboom PE, Westendorp RG: Evidence of genetic enrichment for exceptional survival using a family approach: the Leiden Longevity Study. Eur J Hum Genet 2006;14:79-84.

13 Westendorp RG, van Heemst D, Rozing MP, Frolich M, Mooijaart SP, Blauw GJ, Beekman M, Heijmans BT, de Craen AJ, Slagboom PE: Nonagenarian siblings and their offspring display lower risk of mortality and morbidity than sporadic nonagenarians: the Leiden Longevity Study. J Am Geriatr Soc 2009;57: 1634-1637.

14 Guralnik JM, Simonsick EM, Ferrucci L, Glynn RJ, Berkman LF, Blazer DG, Scherr PA, Wallace RB: A short physical performance battery assessing lower extremity function: association with self-reported disability and prediction of mortality and nursing home admission. J Gerontol 1994;49:M85-M94.

15 Graham J, Ostir G, Fisher S, Ottenbacher K: Assessing walking speed in clinical research: a systematic review. J Eval Clin Pract 2008;14: 552-562.

16 Altmann-Schneider I, van der Grond J, Slagboom PE, Westendorp RG, Maier AB, van Buchem MA, de Craen AJ: Lower susceptibility to cerebral small vessel disease in human familial longevity: the Leiden Longevity Study. Stroke 2013;44:9-14.

17 Altmann-Schneider I, de Craen AJ, Slagboom PE, Westendorp RG, van Buchem MA, Maier $\mathrm{AB}$, van der Grond J: Brain tissue volumes in familial longevity: the Leiden Longevity Study. Aging Cell 2012;11:933-939.

18 Smith SM, Jenkinson M, Woolrich MW, Beckmann CF, Behrens TE, Johansen-Berg H, Bannister PR, De Luca M, Drobnjak I, Flitney DE, Niazy RK, Saunders J, Vickers J, Zhang Y, De Stefano N, Brady JM, Matthews PM: Advances in functional and structural MR image analysis and implementation as FSL. Neuroimage 2004;23(suppl 1):S208-S219.
19 Woolrich MW, Jbabdi S, Patenaude B, Chappell M, Makni S, Behrens T, Beckmann C, Jenkinson M, Smith SM: Bayesian analysis of neuroimaging data in FSL. Neuroimage 2009; 45:S173-S186.

20 Smith SM, De Stefano N, Jenkinson M, Matthews PM: Normalized accurate measurement of longitudinal brain change. J Comput Assist Tomogr 2001;25:466-475.

21 Smith SM, Zhang Y, Jenkinson M, Chen J, Matthews PM, Federico A, De Stefano N: Accurate, robust, and automated longitudinal and cross-sectional brain change analysis. Neuroimage 2002;17:479-489.

22 Boisgontier MP, Beets IA, Duysens J, Nieuwboer A, Krampe RT, Swinnen SP: Age-related differences in attentional cost associated with postural dual tasks: increased recruitment of generic cognitive resources in older adults. Neurosci Biobehav Rev 2013;37:1824-1837.

23 Borel L, Alescio-Lautier B: Posture and cognition in the elderly: interaction and contribution to the rehabilitation strategies. Neurophysiol Clin 2014;44:95-107.

24 Yogev-Seligmann G, Hausdorff JM, Giladi N: The role of executive function and attention in gait. Mov Disord 2008;23:329-342.

25 Soumare A, Tavernier B, Alperovitch A, Tzourio C, Elbaz A: A cross-sectional and longitudinal study of the relationship between walking speed and cognitive function in community-dwelling elderly people. J Gerontol A Biol Sci Med Sci 2009;64:10581065.

26 Montero-Odasso M, Hachinski V: Preludes to brain failure: executive dysfunction and gait disturbances. Neurol Sci 2014;35:601604 .

27 Lundin-Olsson L, Nyberg L, Gustafson Y: 'Stops walking when talking' as a predictor of falls in elderly people. Lancet 1997;349:617.

28 Watson NL, Rosano C, Boudreau RM, Simonsick EM, Ferrucci L, Sutton-Tyrrell K, Hardy SE, Atkinson HH, Yaffe K, Satterfield $S$, Harris TB, Newman AB: Executive function, memory, and gait speed decline in wellfunctioning older adults. J Gerontol A Biol Sci Med Sci 2010;65:1093-1100. 
29 Burke SN, Barnes CA: Neural plasticity in the ageing brain. Nat Rev Neurosci 2006;7:30-40.

- 30 Pasma JH, Stijntjes M, Ou SS, Blauw GJ, Meskers CG, Maier AB: Walking speed in elderly outpatients depends on the assessment method. Age (Dordr) 2014;36:9736.

- 31 Studenski S, Perera S, Patel K, Rosano C, Faulkner K, Inzitari M, Brach J, Chandler J, Cawthon P, Connor EB, Nevitt M, Visser M, Kritchevsky S, Badinelli S, Harris T, Newman AB, Cauley J, Ferrucci L, Guralnik J: Gait speed and survival in older adults. JAMA 2011;305:50-58.

32 Fritz S, Lusardi M: White paper: 'walking speed: the sixth vital sign'. J Geriatr Phys Ther 2009;32:46-49.
33 Thompson CS, Hakim AM: Living beyond our physiological means: small vessel disease of the brain is an expression of a systemic failure in arteriolar function: a unifying hypothesis. Stroke 2009;40:e322-e330.

34 Manor B, Newton E, Abduljalil A, Novak V: The relationship between brain volume and walking outcomes in older adults with and without diabetic peripheral neuropathy. Diabetes Care 2012;35:1907-1912.

35 Sala M, de RA, van den Berg A, AltmannSchneider I, Slagboom PE, Westendorp RG, van Buchem MA, de Craen AJ, van der Grond $\mathrm{J}$ : Microstructural brain tissue damage in metabolic syndrome. Diabetes Care 2014;37:493500 .
36 Arvanitakis Z, Fleischman DA, Arfanakis K, Leurgans SE, Barnes LL, Bennett DA: Association of white matter hyperintensities and gray matter volume with cognition in older individuals without cognitive impairment. Brain Struct Funct 2015, Epub ahead of print.

37 Silbert LC, Nelson C, Howieson DB, Moore MM, Kaye JA: Impact of white matter hyperintensity volume progression on rate of cognitive and motor decline. Neurology 2008;71: 108-113.

38 Atiya M, Hyman BT, Albert MS, Killiany R: Structural magnetic resonance imaging in established and prodromal Alzheimer disease: a review. Alzheimer Dis Assoc Disord 2003;17: 177-195. 